\title{
Transmission of MIMO - OFDM signal using Optical Link
}

\author{
Shikha Nema ${ }^{\# 1}$, Snehal Gaikwad ${ }^{* 2}$ \\ ${ }^{\#}$ Professor, Mumbai University, " M.E. Scholar, Mumbai University
}

\begin{abstract}
Wireless networks have quickly become part of everyday life. Wireless LANs, cell phone networks, and personal area networks are just a few examples of widely used wireless networks. However, wireless devices are range and data rate limited. The research community has spent a great deal of effort on finding ways to overcome these limitations. In other words, high Data-Rate wireless access is demanded by many applications. Traditionally, more bandwidth is required for higher data-rate transmission. However, due to spectral limitations, it is often impractical or sometimes very expensive to increase bandwidth. Multiple transmit and receive antennas can be used to form multiple-input multiple-output (MIMO) channels to increase the capacity by a factor of the minimum number of transmit and receive antennas. Along with MIMO channels Orthogonal frequency division multiplexing (OFDM) i.e. MIMO-OFDM is considered for wideband transmission to mitigate inter symbol interference and enhance system capacity, Whereas optical communication has several benefits such as larger bandwidth, reduced power consumption that has made it an attractive implementation option for various communication systems including wireless LAN's. This paper investigates the feasibility of MIMO-OFDM technique optical fiber based system i.e. MIMO-OFDM for optical communication.
\end{abstract}

Keywords:- Fast Fourier Transform(FFT), Inverse Fast Fourier Transform(IFFT), Multiple Input Multiple Output (MIMO), Orthogonal Frequency Division Multiplexing (OFDM).

\section{INTRODUCTION}

The performance of wireless communication systems is mainly governed by the wireless channel environment. As opposed to the typically static and predictable characteristics of a wired channel, the wireless channel is rather dynamic and unpredictable, which makes an exact analysis of the wireless communication system often difficult. In recent years, optimization of the wireless communication system has become critical with the rapid growth of mobile communication services and emerging broadband mobile Internet access services. In fact, the understanding of wireless channels will lay the foundation for the development of high performance and bandwidth-efficient wireless transmission technology [2]. The requirement for wide bandwidth and flexibility imposes the use of efficient transmission methods that would fit to the characteristics of wideband channels especially in wireless environment where the channel is very challenging.

In wireless environment the signal is propagating from the transmitter to the receiver along number of different paths, collectively referred as multipath. While propagating the signal power drops of due to three effects: path loss, macroscopic fading and microscopic fading. Fading of the signal can be mitigated by different diversity techniques. To obtain diversity, the signal is transmitted through multiple independent fading paths e.g. in time, frequency or space and combined constructively at the receiver. Multiple input multiple output (MIMO) exploits spatial diversity by having several transmit and receive antennas [3]. OFDM is modulation method known for its capability to mitigate multipath as well as inter symbol interference. In OFDM the high speed data stream is divided into Nc narrowband data streams, $\mathrm{Nc}$ corresponding to the subcarriers or sub channels i.e. one OFDM symbol consists of $\mathrm{N}$ symbols modulated for example by QAM or PSK.[4].

The demand for broadband services has driven research on millimeter- wave frequency band communications for wireless access network due to its spectrum availability, and compact size of radio frequency devices. The mmwave signals suffer from severe loss along the transmission as well as atmospheric attenuation. Optical fiber is a technology by which microwave (electrical) signals are distributed by means of optical components and techniques. One of the major motivation and system requirements for optical fiber technology is reduced complexity of the transmission equipment close to the antennas, a minimised visual impact of these equipments, and lower operational costs of the network due to infrastructure sharing among different Base Stations.

The electrical signal distributed may be baseband data, modulated IF, or the actual modulated RF signal. The electrical signal is used to modulate the optical source. The resulting optical signal is then carried over the optical fiber link to the remote station. By delivering the radio signals directly, the optical fiber link avoids the necessity to generate high frequency radio carriers at the antenna site. Since antenna sites are usually remote from easy access, there is a lot to gain from such an arrangement. However, the main advantage of optical fiber is the ability to concentrate most of the expensive, high frequency equipment at a centralized location, thereby making it possible to use simpler remote sites. The critical advantage of using OFDM in optical fiber communications includes better spectral efficiency, elimination of sub channel and symbol interference using the fast Fourier transform (FFT) for modulation and demodulation, which does not require any equalization and dispersion tolerance. Optical fiber is very attractive technique for wireless access network infrastructure, 
because it can transmit microwaves and milli meterwaves over a long distance [1].

The combination of these three technology i.e MIMO, OFDM and Optical fiber can overcome the obstacles in wireless communication and transmit the signal with high throughput, high data rate and high bandwidth.

\section{MIMO-OFDM}

\section{A. MIMO System \& Model}

Multiple input multiple output (MIMO) techniques employing multiple antennas at both ends of a transmission link have received attention because of the potential improvements in data transmission rates and link reliability [8]. The fig. 1 shows the reference model of MIMO system with $\mathrm{n}$ number of transmitting and receiving antenna.

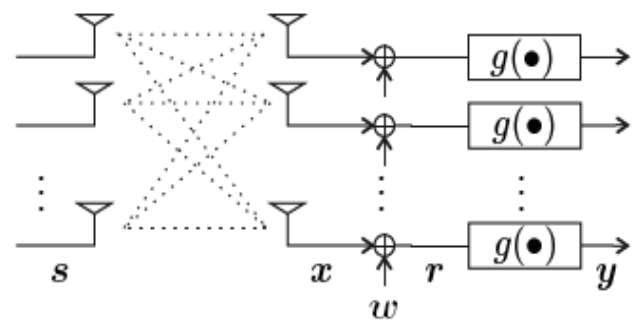

Fig.1 MIMO Reference Model [5]

The transmitter emits the signal s $\in \mathrm{C}^{\mathrm{NT}}$ over stationary memory less flat fading channel $\mathrm{H} \in \mathrm{C}^{\mathrm{NR} \times \mathrm{NT}}$ with tapgain $\mathrm{h}_{\mathrm{ij}}$ from the $\mathrm{j}^{\text {th }}$ transmit to the $\mathrm{i}^{\text {th }}$ receive antenna. Block fading model is used for the channel $\mathrm{H}$. The channel remains constant for some period, long enough to allow for accurate estimation, and changes to an independent realization in the next block. This can be achieved with a sufficiently long interleaver. The received vector $\mathrm{x} \in \mathrm{C}^{\mathrm{NR}}$ is perturbed by a zero mean circularly symmetric Gaussian noise vector w $\in C^{\mathrm{NR}}$. A function $\mathrm{g}(\cdot)$ acts on every antenna, producing the output vector as,

$$
\mathrm{y}=\mathrm{g}(\mathrm{r})=\mathrm{g}(\mathrm{x}+\mathrm{w})
$$

The function $\mathrm{g}$ (.) maps the complex valued received signal $r$ from the complex plane into one real dimension, element-wise on every antenna. The detector has access only to $\mathbf{y}[6]$.

Let, $\mathrm{h}_{\mathrm{mn}}$ be a complex number corresponding to the channel gain between transmit antenna $n$ and receive antenna $\mathrm{m}$. If at a certain time instant the complex signals $\left\{\mathrm{x}_{1}, \ldots, \mathrm{x}_{\mathrm{NT}}\right\}$ are transmitted via the $\mathrm{N}_{\mathrm{T}}$ antennas, respectively, the received signal at antenna $\mathrm{m}$ can be expressed as

$$
\mathrm{y}_{\mathrm{m}}=\sum_{\mathrm{n}=1}^{\mathrm{N}_{\mathrm{T}}} \mathrm{h}_{\mathrm{mn}}+\mathrm{w}_{\mathrm{m}}
$$

Where, $\mathrm{w}_{\mathrm{m}}$ is a noise term.

The above relation can be easily expressed in a matrix framework. Let $\mathrm{x}$ and $\mathrm{y}$ be $\mathrm{N}_{\mathrm{T}}$ and $\mathrm{N}_{\mathrm{R}}$ vectors containing the transmitted and received data, respectively. Define the following $\mathrm{N}_{\mathrm{T}} \times \mathrm{N}_{\mathrm{R}}$ channel gain matrix

$$
\mathrm{H}=\left[\begin{array}{ll}
\mathrm{h}_{1,1} & \mathrm{~h}_{1, \mathrm{NT}} \\
\mathrm{h}_{\mathrm{NR}, 1} & \mathrm{~h}_{\mathrm{NR}, \mathrm{NT}}
\end{array}\right]
$$

Then, the received signal can be written as

$$
\mathrm{y}=\mathrm{Hx}+\mathrm{w}
$$

Where, $w=\left[w_{1} \cdots w_{\mathrm{NR}}\right]^{T}$ is a vector of noise samples. If several consecutive vectors $\left\{\mathrm{x}_{1}, \ldots, \mathrm{x}_{\mathrm{N}}\right\}$ are transmitted, the corresponding received data can be arranged in a matrix

$$
\mathrm{Y}=\left[\mathrm{y}_{1} \cdots \mathrm{y}_{\mathrm{N}}\right]
$$

and written as follows:

$$
\mathrm{Y}=\mathrm{HX}+\mathrm{W}
$$

Where, $\mathrm{X}=\left[\mathrm{x}_{1} \cdots \mathrm{x}_{\mathrm{N}}\right]$ and $\mathrm{W}=\left[\mathrm{w}_{1} \cdots \mathrm{w}_{\mathrm{N}}\right]$.

\section{B. OFDM system \& Model}

A multi-carrier communication system with orthogonal sub-carriers is called an Orthogonal Frequency Division Multiplex (OFDM) system. In an OFDM system, the carrier spacing $\Delta \mathrm{f}$ is $1 / \mathrm{NT}$, where $\mathrm{N}$ is the number of the carriers and $1 / \mathrm{T}$ is the overall symbol rate.
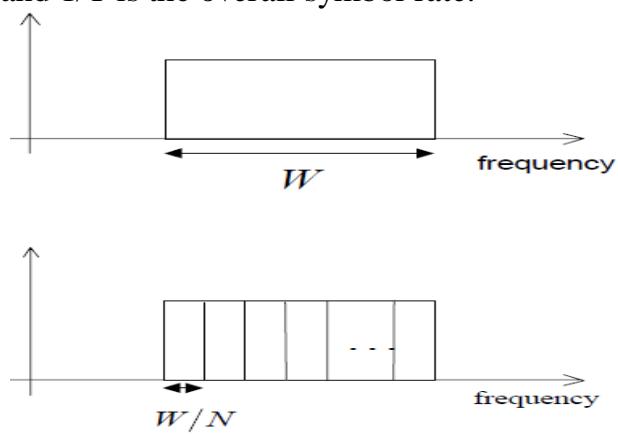

Fig.2 Multicarrier System[10]

With this carrier spacing, the sub-channels can maintain orthogonally, although the sub-channels overlap. Therefore, there is no inter-sub-carrier interference with ideal OFDM systems. The number of sub-carriers $\mathrm{N}$ is chosen so that the sub-channel bandwidth is less than the channel coherence bandwidth. Under this condition, each sub-channel does not experience significant Inter-symbol Interference (ISI)

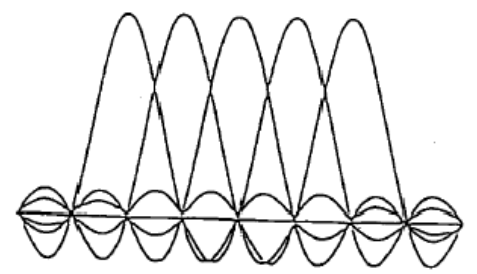

Fig.3 OFDM Spectrum [10]

In the receiver the pilot symbol is used for synchronization at the starting stage and the same symbol is used for equalization at the later stage. All the other 
processes at the receiver are the reverse process of the transmitter. The mathematical equivalent of OFDM signal is expressed as

$$
\mathrm{v}(\mathrm{t})=\sum_{\mathrm{k}=0}^{\mathrm{N}-1} \mathrm{X}_{\mathrm{k}} \mathrm{e}^{\mathrm{j} 2 \pi \mathrm{kt} / \mathrm{T}} 0 \leq \mathrm{t}<\mathrm{T}
$$

where, $X_{k}$ are the data symbols and $T$ is the OFDM symbol time. The sub-carrier spacing of $1 / \mathrm{T}$ makes them orthogonal over each symbol period [7].

\section{MIMO-OFDM system \& Model}

MIMO-OFDM combines OFDM and MIMO techniques thereby achieving spectral efficiency and increased throughput. A MIMO-OFDM system transmits independent OFDM modulated data from multiple antennas simultaneously. At the receiver, after OFDM demodulation, MIMO decoding on each of the subchannels extracts the data from all the transmit antennas on all the subchannels. In this application MIMO-OFDM combines the advantages of MIMO with tolerance to delay spread. OFDM system can exploit different MIMO approaches in order to enhance the data throughput or transmission reliability. These techniques include STC [9], spatial multiplexing, beam forming, and some combinations of these. These techniques can, for example, are classified according to whether they are applied in the frequency or time domain, at the receiver or at the transmitter, with closely located antennas or separate antennas, or for example whether they need to know the channel state information i.e. channel matrix at the transmitter or not.

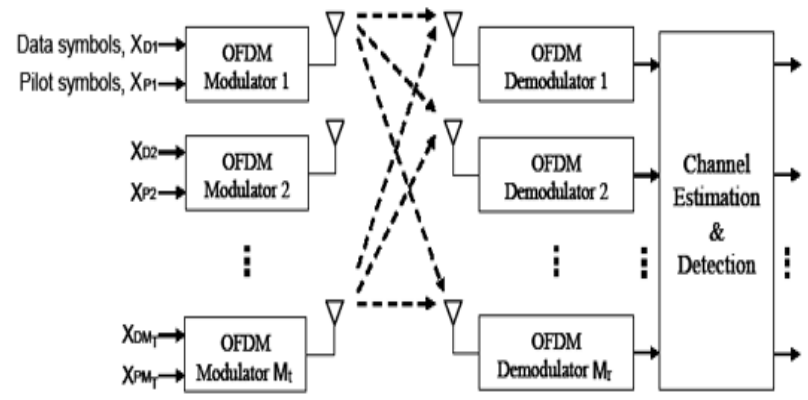

Fig.4 MIMO-OFDM System

The general transceiver structure of MIMO-OFDM is presented in Fig.4. The system consists of $\mathrm{N}$ transmit antennas and $\mathrm{M}$ receive antennas. The cyclic prefix is assumed to be longer than the channel delay spread. The OFDM signal for each antenna is obtained by using inverse fast Fourier transform (IFFT) and can be detected by fast Fourier transform (FFT).

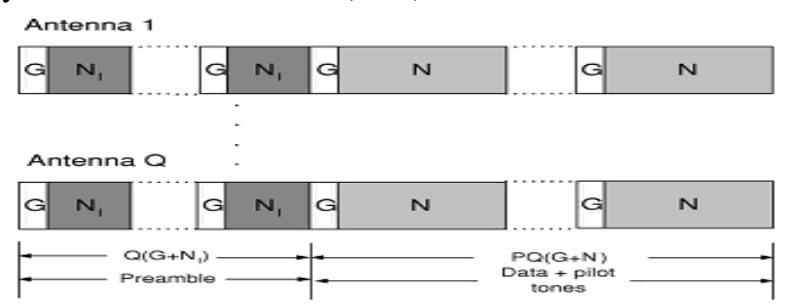

The frame structure of a typical MIMO-OFDM system is shown in Fig.5. The OFDM preamble consists of $Q$ training symbols of length $N_{I}+G$, where, $G \leq N_{I} \leq N$, $\mathrm{N}_{\mathrm{I}}=\mathrm{N} / \mathrm{I}$ and $\mathrm{I}$ is an integer that divides $\mathrm{N}$. Often the length of the guard interval in the training period is doubled. Consider the preamble portion of the OFDM frame. The length $\mathrm{N}_{\mathrm{I}}+\mathrm{G}$ preamble sequences are obtained by exciting every $\mathrm{I}^{\text {th }}$ coefficient of a length- $\mathrm{N}$ frequency-domain vector with a nonzero training symbol from a chosen alphabet.

\section{MIMO-OFDM FOR OPTICAL COMMUNICATION}

The increasing demand for higher data rates in wireless broadband services is pushing towards smaller cell sizes and a higher number of Base Stations. In this situation, the use of optical fibers is an efficient solution enabling infrastructure sharing and operational expenses reduction. Several scenarios where RoF links could provide compelling advantages, for example in WiMAX infrastructure rollouts, have been identified for e.g. Optically fed distributed antenna systems, where an assortment of low power indoor remote antenna units are fed by WiMAX over fiber links from a central location or control station.

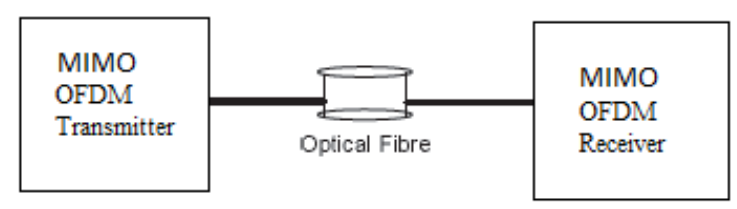

Fig. 6 MIMO-OFDM with Optical Fiber link

The modal dispersion of multimode fiber can be exploited by MIMO rather than being avoided [12]. Note that for a long time the multipath nature of wireless channels was viewed as a limiting factor to be avoided. In recent years it has been realized that the multipath nature of a channel can actually enhance throughput if it is properly exploited. If each guiding mode is regarded as a scattering path, MMF behaves similar to a wireless channel with rich multipath scattering which is shown in the fig.7.

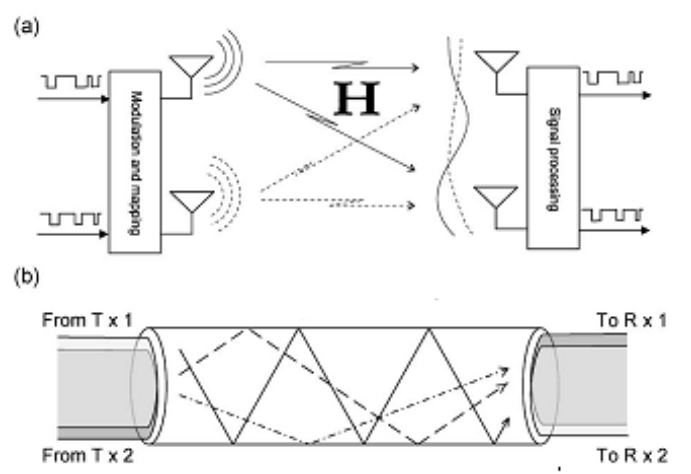

Fig.7.a) wireless channel b) Multimode channel[12]

Fig.5 Frame Structure [11] 
The multi mode fiber is simulated using impulse response model. To model optical fiber channel as a linear system therefore, for an intensity modulated system we can express the output intensity spectrum $\mathrm{I}_{0}(\mathrm{f})$ of the channel in terms of the input intensity spectrum $\mathrm{I}_{\mathrm{i}}(\mathrm{f})$ as,

$$
I_{0}(f)=|H(f)|^{2} I_{i}(f)
$$

Where, $\mathrm{H}(\mathrm{f})$ is the field transfer function from the input to output field amplitude for the fiber channel.

The magnitude squared of the field transfer function is required in order to relate the input field intensity spectrum to the output field intensity spectrum. Therefore, we write, field intensity transfer function in terms of normalized frequency as ,

$$
\begin{aligned}
& |\mathrm{H}(\mathrm{f})|^{2}=\left\{\left(((2-\delta) / 2) \cos (\delta-2)^{2} \mathrm{f}\right) \cdot \sin \mathrm{c}((2-\delta) \mathrm{f})\right. \\
& \left.+\left(2 \pi \delta^{2} / 64 \mathrm{f}\right)\left[\mathrm{C}\left(\xi_{1}\right)+\mathrm{C}\left(\xi_{2}\right)\right]\right\}^{2} \\
& +\left\{\left(( 2 - \delta / 2 ) \operatorname { s i n } \left((\delta-2)^{2} \mathrm{f} \sin \mathrm{c}((2-\delta) \mathrm{f})\right.\right.\right. \\
& \left.+\left(2 \pi \delta^{2} / 64 \mathrm{f}\right)\left[\mathrm{S}\left(\xi_{1}\right)+\mathrm{S}\left(\xi_{2}\right)\right]\right\}^{2}
\end{aligned}
$$

where,

$$
\begin{gathered}
\xi_{1}=\left(\delta^{2} \mathrm{f} / 2 \pi\right)^{1 / 2} \\
\xi_{2}=\left[\left(\left(\delta^{2}-4\right)^{2} \mathrm{f} / 2 \pi\right)^{1 / 2}\right]
\end{gathered}
$$

$\delta$ is dispersion parameter and value of it varies in between 0 and 4.

Frequency $\mathrm{f}$ is given by,

$$
\mathrm{f}=\Delta^{2} \mathrm{~T}_{0} \pi \mathrm{f} / 4
$$

where, $\Delta$ is given by

$$
\Delta=\frac{\mathrm{n}_{0}^{2}-\mathrm{n}_{\mathrm{cl}}^{2}}{2 \mathrm{n}_{\mathrm{cl}}^{2}}
$$

where, $\mathrm{n}_{0}$ is refractive index of core, $\mathrm{n}_{\mathrm{cl}}$ is refractive index of clad and $\mathrm{T}_{0}$ is given by

$$
\mathrm{T}_{0}=\frac{\operatorname{Ln}_{0}}{\mathrm{c}}
$$

where, $\mathrm{L}$ is length of fiber, $\mathrm{c}$ is velocity of light.

The above transfer function is used for modeling of multimode optical link.

These equations are used for the performance analysis of optical fiber.

\section{SIMULATION RESULTS}

The system is modeled using Matlab to allow various parameters of the system to be varied and tested. The aim of doing the simulations is to measure the performance of MIMO-OFDM under different modulation techniques and to allow for different configurations of MIMO to be tested.

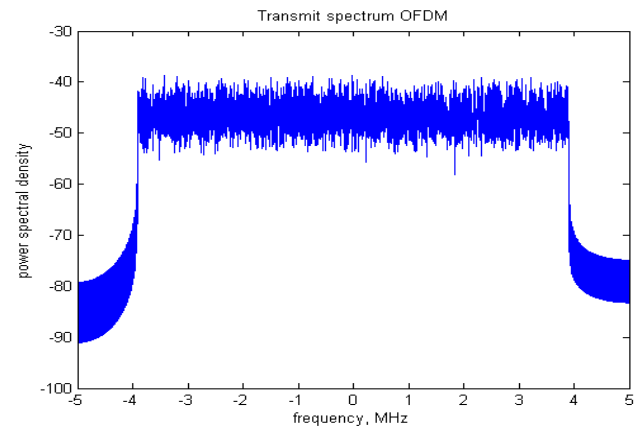

Fig. 7 OFDM Spectrum

In fig.7, the OFDM signal spectrum can be seen corresponding to a random binary input sequence.

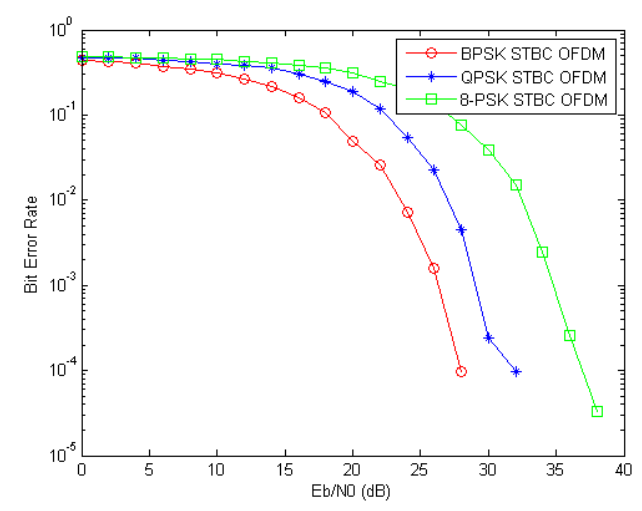

Fig.8 MIMO-OFDM with2x2

Fig.8 illustrates the BER performance of MIMO-OFDM system with 2 transmitting and 2 receiving antenna using BPSK, QPSK \& 8-PSK signaling. It can be observed that as signal to noise ratio increases bit error rate decreases. As well as if number of bits per symbol increases the bit error rate increases. So we have to compromise on the symbol period and noise that can be handle by the receiver.

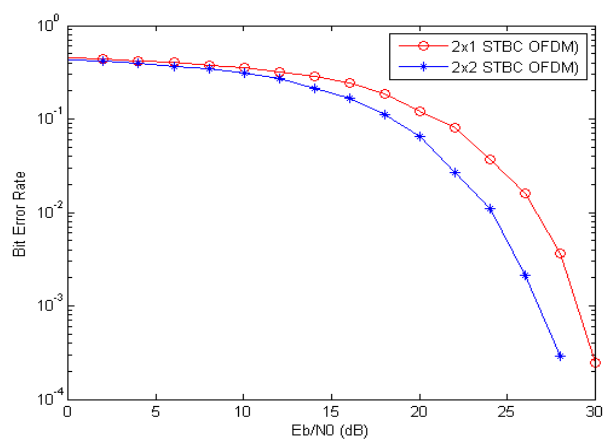

Fig.9. BER Vs SNR for $2 \times 1$ and $2 \times 2$ system

Fig.9 illustrates the BER performance of MIMO-OFDM system with 2 transmitting and 1 receiving antennas \& 2 transmitting and 2 receiving antennas. It shows that the BER performance improves with increase in number of antennas at the transmitting and receiving side. Bit error rate is less for $2 \times 2$ system as compare to $2 \times 1$ system. 


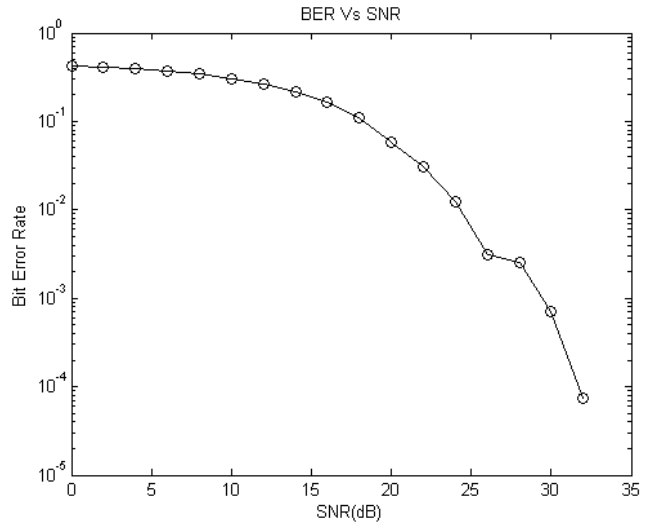

Fig.10 2x2 MIMO-OFDM without optical link

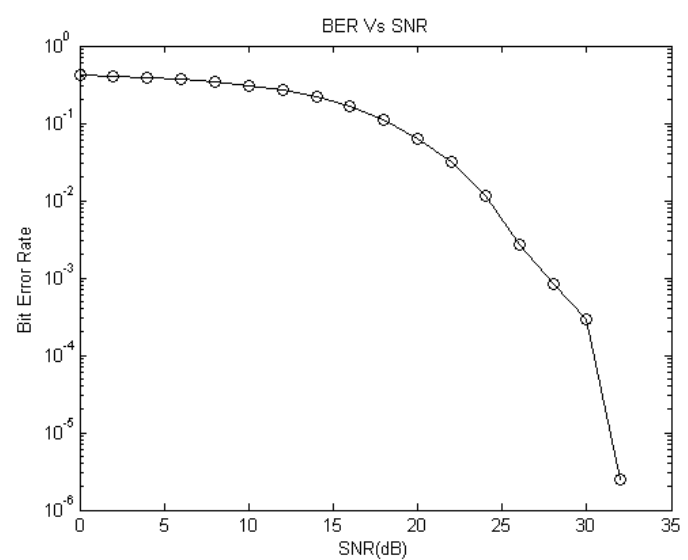

Fig.11 2x2 MIMO-OFDM with optical link

Fig.10 shows the MIMO-OFDM system with 2 transmitting and 2 receiving antenna without optical link whereas fig. 11 shows with optical system. From this it can observe that the BER performance of the system with optical link improves than without optical link. The BER for without optical system is $10^{-4}$ whereas for with optical system is $10^{-6}$.

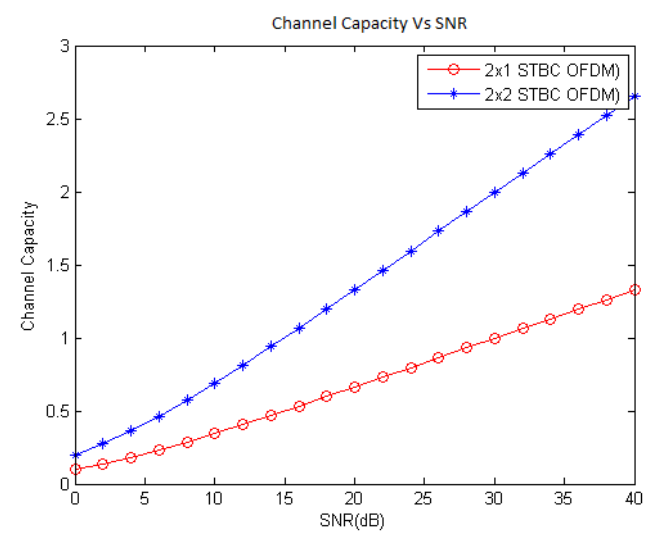

Fig. 12 Channel capacity for $2 \times 1$ and $2 \times 2$

From fig. 12 shows the graph of channel capacity Vs signal to noise ratio. Channel capacity is the maximum data rate that can be carried over the transmission medium. So from the graph it can be analysed that with increasing the SNR at low values capacity is increasing linearly, while going higher value of SNR capacity increases logarithmically.

\section{Conclusions}

The studying of OFDM modulation and radio over fiber technology becomes very important because both of them have been developed to support some important future wireless systems. This paper gives the study of performance analysis of RoF system for transmission of MIMO - OFDM based signals for long distance transmission. MIMO - OFDM is a very promising technology for optical communications, but the very different constraints introduced open up many new interesting avenues for research.

\section{REFERENCES}

[1] Ahmad Said Chahine, Uche A. K Okon kwo and Razali Ngah "Study the Performance of OFDM Radio over Fiber for Wireless Communication Systems", IEEE international conference on RF and microwave, proceeding, Dec 2008

[2] Gurphh sin \& sind Arokiaswami Alphones "OFDM Modulation Study for a Radio-over- Fiber System for Wireless LAN (IEEE 802.11a)",School of Electrical and Electronic Engineering Nanyang Technological University, Singapore 639798.

[3] A.J. Paulraj, D.A. Gore, R.U. Nabar, H. Bolcskei, "An overview of MIMO communications - a key to gigabit wireless" Proceedings of the IEEE, Volume: 92, Issue: 2, Feb. 2004 Pages: $198-218$

[4] Jean Armstrong "OFDM for Next Generation Optical Communicatio Systems", IEEE Proceedings of con 2008

[5] G. K. Psaltopoulos, F. Trösch, and A. Wittneben, "On achievable rates of MIMO systems with nonlinear receivers," in Proc. IEEE International Symp. Inf. Theory, June 2007, pp.1071-1075.

[6] Georgios K. Psaltopoulos, Armin wittneben, "Nonlinear MIMO: Affordable MIMO technology for wireless sensor networks" IEEE transaction on wireless communication, Vol 9, No.2, Feb 2010.

[7] Jean Armstrong "OFDM for optical communication", Journal of lightwave technology, vol. 27, no. 3, February1, 2009.

[8] I. E. Telatar, "Capacity of multiantenna Gaussian channels", Eur. Trans. on Telecommun (ETT), vol. 10, pp. 585595, Nov. Dec. 1999.

[9] V. Tarokh, N. Seshadri, and A.R. Calderbank, "Spacetime codes for high data rate wireless communications: performance criterion and code construction," IEEE Trans. Inform. Theory, vol. 44, no. 2, pp. 744765, March 1998.

[10] W. Y. Zou and Y. Wu, "COFDM: An overview," IEEE Trans. Broadcasting, vol. 41, pp. 1-8, 1995.

[11] Gordonl Stuber, John R. Barry "Broadband MIMOOFDM Wireless Communications" Proceeding of the IEEE, vol. 92, No.2, Feb,2004.

[12] Alireza Tarighat, Rick C. J. Hsu, Akhil Shah, "Fundamentals and Challenges of Optical MultipleInput Multiple-Output Multimode Fiber Links" IEEE Communications Magazine, May 2007. 